\title{
Designing AR Game Enhancing Interactivity between Virtual Objects and Hand for Overcoming Space Limit
}

\author{
Kyungyeon Moon, Jonghee Sang, and Woontack Woo \\ KAIST Graduate school of Culture Technology, Daejeon 305-701, S. Korea \\ \{moonjeje, jhsang21, wwoo\} @kaist.ac.kr
}

\begin{abstract}
We propose real-time interactive game that is based on Augmented Reality (AR). It is composed of AR marker, Head Mounted Display and depth camera. By using marker, the proposed system augments game space, fishing place. And player can interact virtual game object such as bait or fish with bare hands based on computer vision. The rapid development of AR technologies has raised profound interests in the design of AR games, but the existing games have not provided realistically felt game environments because the way to play games remains the same when the platform is changed. In addition, studies in this field did not fully utilize AR technologies, so that inherent characteristics of AR game do not impact user experience and draw attention explicitly on design concepts. Our system gives the experience that is grasping the virtual objects. Also, it can be applied to various game contents that are actually felt as real.
\end{abstract}

Keywords: entertainment, augmented reality, 3D interaction, HMD, handtracking.

\section{Introduction}

\subsection{Background}

User interface design environment has been known to be a part of significant elements in game system.[1] Nowadays, traditional game interfaces such as joysticks, mouse and keyboards are shrinking every year[2] and game system starts to build AR basedinterface.[3] These new streams of game interface design have brought some changes which are spatial transformation. For example, people can play the game while they are walking. And game environments escape from 2D into 3D. It means that game space expands out of monitors. Also, players expect to get a new experience they cannot get from other existing or similar form of games.[4]

\subsection{Problem-Posing}

With respect to players' demand, game console makers have made effort to mix the reality and virtual reality. So Nintendo 'Wii', Sony 'Move' and Microsoft 'Kinect' are developed.[5] Developed AR games were based on motion recognition technologies.[6] 
However, there are some problems. First, these devices can recognize big motions like dancing or boxing. Thus, in small residential space or public place such as apartment and café, people have trouble with using it. Also, people who are wearing heavy clothes or skirts that hide body parts cannot use it because the devices cannot recognize gamers.[7] Therefore, small motion or elaborate control has difficulty handling the system embedded in these devices. Third problem is game controllers. Existing system needs to have traditional game controllers, which means that if people sit far from game devices, they cannot play with it. In addition, if users want to play tennis or guitar game, they should buy tennis racket controller or guitar controller that is suitable for specific device.[8] Finally, gamers cannot interact with game objects in $3 \mathrm{D}$ space. The game objects remain to be still locked in the monitor.

\subsection{System Overview}

Our system provides players with distinguished experience that users can get into game space. We made fishing game and developed all the components needed to play the game in AR. A player can fish by virtual baits and virtual hook that are controlled by player's hands and motion.[9] It means that they can touch and interact with augmented objects using bare hands. Fig.1 shows the game space which is built by our system.

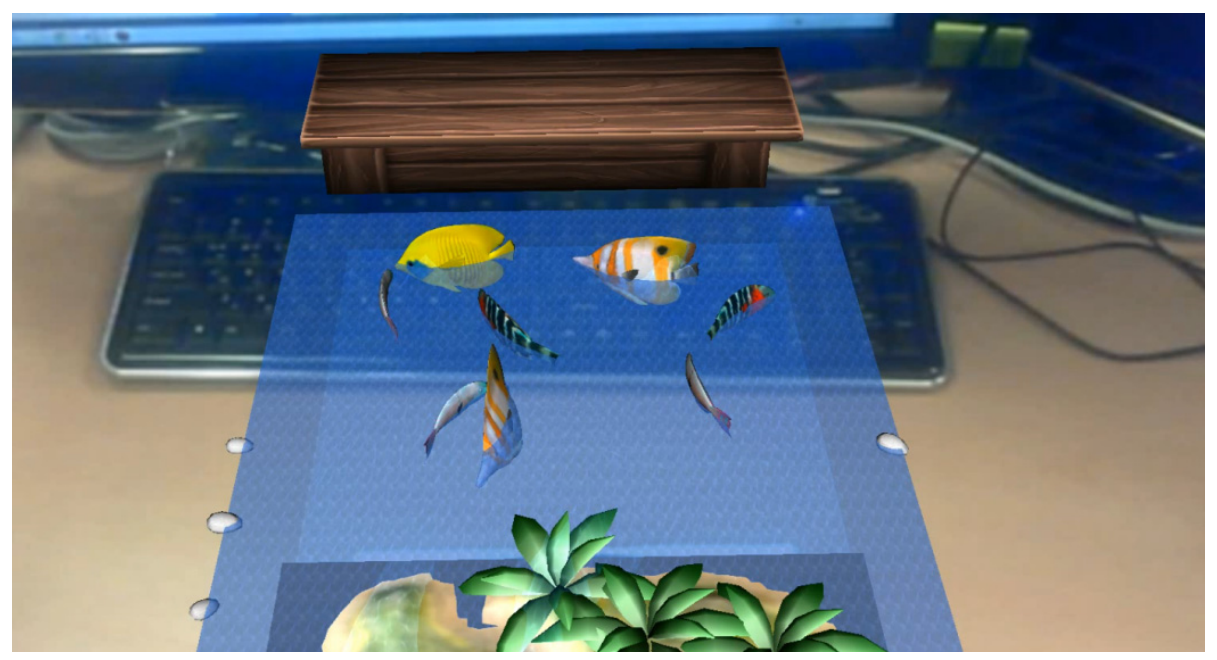

Fig. 1. Scene of our system, AR fishing game scene that user saw through a HMD. We developed the game environment and all objects by Unity.

\section{System Design}

\subsection{Game Scenario}

Fishing in real world is an outdoor activity. For giving users realistic-looking fishing place, we made game space in $3 \mathrm{D}$ not $2 \mathrm{D}$ which is shown as Fig.1. If users have 
HMD and the marker, they can face the virtual fishing place anywhere. Fig.2 shows summary of the system scenario.

We replicated similar interactions, which existed in real fishing techniques. These interactions are controlling the system. For example, the motion of harpooning is used to catch fish in our system. There are some techniques for catching fish; hand gathering, spearing, netting, angling and trapping. Among these techniques, we focused on implementing spear fishing, which is an ancient method of fishing that has been used throughout the world. So, it is intuitive for playing and user can catch how to play more quickly.[10]

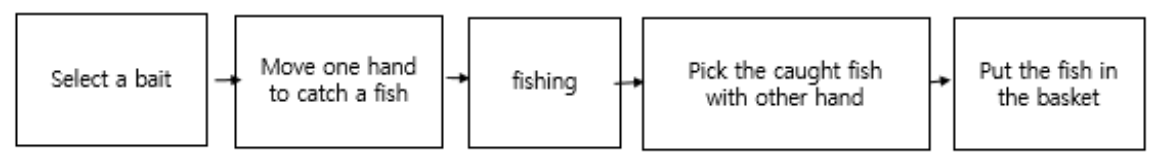

Fig. 2. The game scenario. These steps progress with a user wearing a HMD.

\subsection{Game Flow}

As we designed the following interactions, our system aims to provide a user with immersion. (a) When user sees the marker, the game space that is virtual fishing hole is augmented on real space. And then (b) user opens his/her palm in front of HMD, system is ready to start the AR fishing game. After that, fishing tool like rod or spear is also augmented at user's fingertip. According to [11], it is possible to recognize hand tracking in real time. So, (c) user can catch fish directly with bare hands where the virtual fishing tool hung, and put it in the basket.

We put some entertaining and sport fishing elements. For pleasure and competition, we add recreational element, which is a time limit. Player should catch fish as many as possible within limited time.

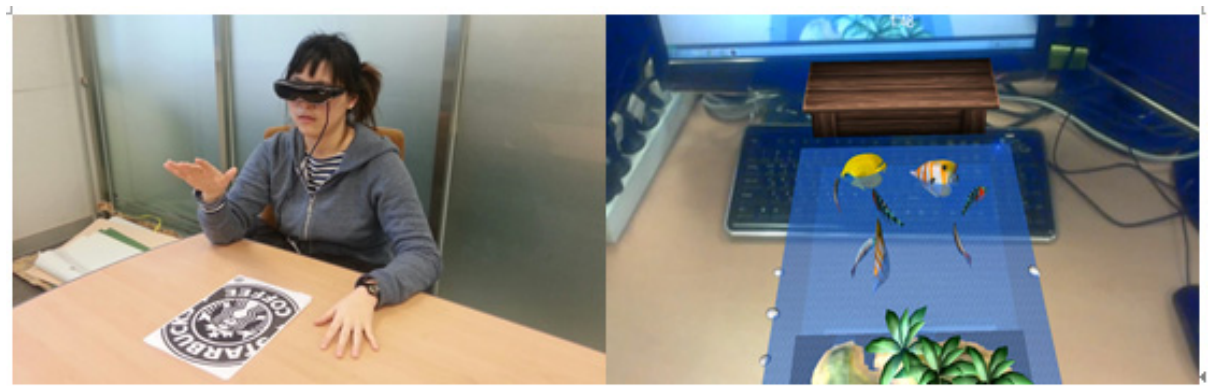

Fig. 3(a). See the marker through HMD. Left is the scene of seeing the marker. Right is the scene of game space. All objects were augmented. 


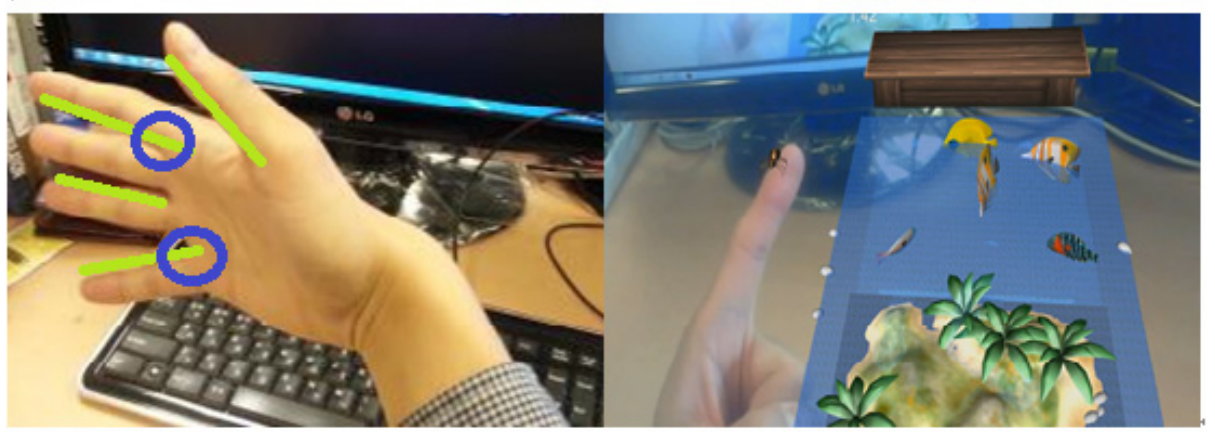

Fig. 3(b). Recognize the hands. The camera recognizes the position of tip of fingers.

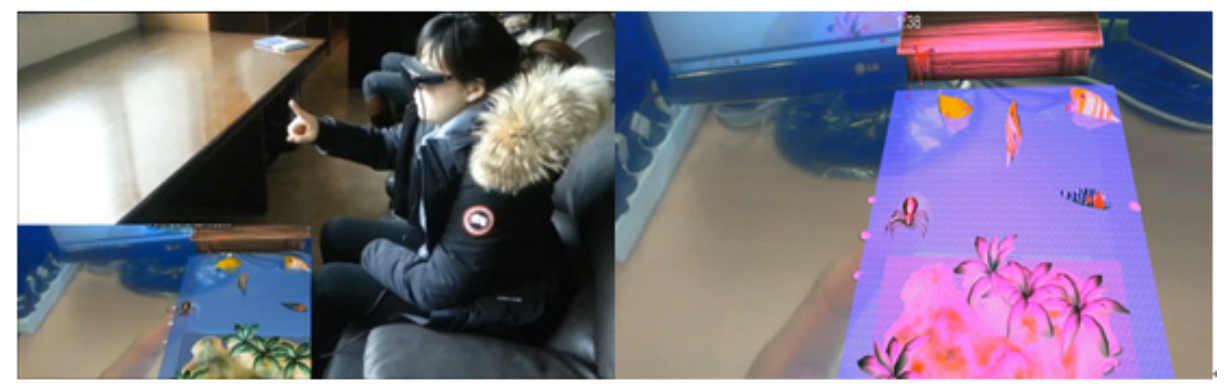

Fig. 3(c). Play the game. User can play the system anywhere.

\section{Implementation}

\subsection{Hardware}

Our augmented reality system is based on a window7. We required RGB-D camera and HMD. HMD is Accupix Mybud with $852 \times 480$ pixel high resolution in each eye and a horizontal field of view of approximately $35^{\circ}$ and Intel creative gesture camera as RGB-D camera which is $1280 \times 720$ pixel high resolution and depth resolution is QVGA $(320 \times 240)$. The software was written in a C\# using Vuforia SDK in Unity3D.

\subsection{Flow of System}

The proposed system is divided into four procedures, capturing RGB-D image, setting the environment, hand gesture recognition, and rendering as seen from Fig.4. 


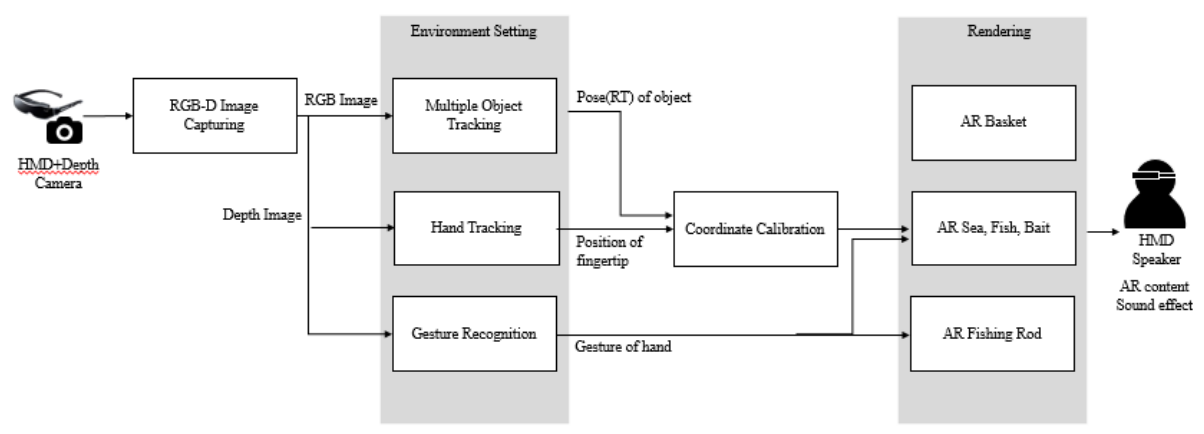

Fig. 4. System Flow chart

\subsection{Interaction Techniques}

Targeting. Vuforia supports sophisticated algorithms to detect and track the features. Vuforia SDK recognizes the image target by comparing these natural features against a known target resource database.[12] When the image is detected, the environment of the simulation is appeared onto the image.

The overall pattern of data flow within the software is shown in Fig.4. Recall that Intel creative gesture camera as RGB-D camera. From the data from RGB-D camera, we can exploit various kinds of interaction.[13] First, we can use the image targeting to create a coordinate frame for the image, and then we make the fish environment for the user. Second, we can use the hand tracking data to create a coordinate frame for the hand, and track the hand point direction. We can orient both of these coordinate frame in to the same sense( $\mathrm{Y}$ is up, $\mathrm{X}$ to the right, $-\mathrm{Z}$ in to the screen or away from the user and roll is rotation around $\mathrm{Z}$, pitch around $\mathrm{X}$, yaw around $\mathrm{Y}$ ).

Tracking. The hand is recognized through camera. The user can move freely inside the interaction area in front of the Creative Interactive Gesture Camera. The tracking itself is accomplished by one camera system attached on the HMD. The visualizations is based on the camera system and rendered in HD quality on a HMD. The following paragraphs explain the components:

Tracking of the hand was only needed for viewpoint. We selected a pragmatic and inexpensive solution with a Creative Interactive Gesture Camera that detects the nearest hand in front of camera. The viewpoint (the virtual camera in the 3D world) is moved accordingly to provide an immersive depth cue. Additionally, for selected levels the user is moved to another position in the 3D world. [14]

For more natural motion, we removed controller which is disturbing immersion for the sense of realism. Without additional cumbersome supplementary devices, users can gain the use of naturalness while they enjoyed the game. 


\section{Evaluation}

\subsection{Objective}

The objective of this study was to build system that users would interact with virtual objects in 3D space using bare hands. For testing effectiveness and usefulness of our system, which estimates how comfortable users use and how easy users play, we should measure what users feel by qualitative and quantitative methods. Then, we analyze the result and adjust the system for maximize user satisfaction and immersion.

\subsection{Procedure}

24 colleagues $(12 f+12 m$, aged $22-28$, students of graduate school of cultural and technology) evaluated our system. No one had previously experience with fish game with HMD. Thus, we gave a short introduction $(3 \mathrm{~min})$ for all participants. This includes watching the video $(1 \mathrm{~min})$ and trying one practice game for each participant.

Qualitative Measure. We collected participant comments in a post-experiment interview to gain further information. Also, making a questionnaire, we hand out a questionnaire to 24 subjects. The questionnaire is in Fig.5. The odd number of question is about ease of playing and even is about difficulty of playing. If subjects agree strongly, scoring 5 points, or else scoring 0 point.

Quantitative Measure. The quantitative measures were experiments consisted of estimating the number of fish users caught during the tests in limited time, which is in 2 minutes. This experiment was used to adjust system composition. For example, it would help us determine the most suitable size of augmented objects.
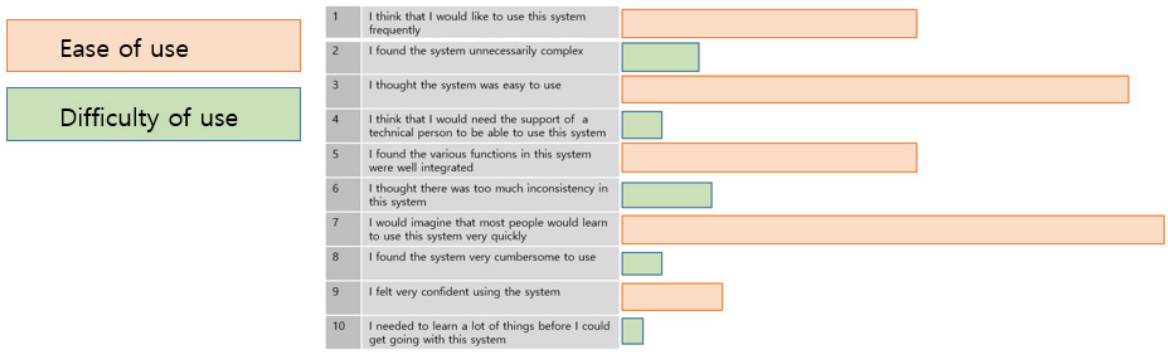

Fig. 5. The questionnaire and result 


\subsection{Result and Analysis}

The results of the questionnaire are summarized in Fig.5. As result shows, most people strongly agreed that people understand how to use it easily (95.8\%). The question that gains the lowest point is about people can acquire something before using this system. It means that people think almost nothing to be learned such as tutorial or mechanisms of some controllers when playing our system. However, 22 subjects responded to the question 'is it easy?' with strongly agreement.

Results of experiments show in Fig.6. Subjects fished 4.8 fish averagely within 2 minutes. All subjects caught at least 3 fish. $25 \%$ of subjects clear the game. So the degree of difficulty in system needed to be modified.

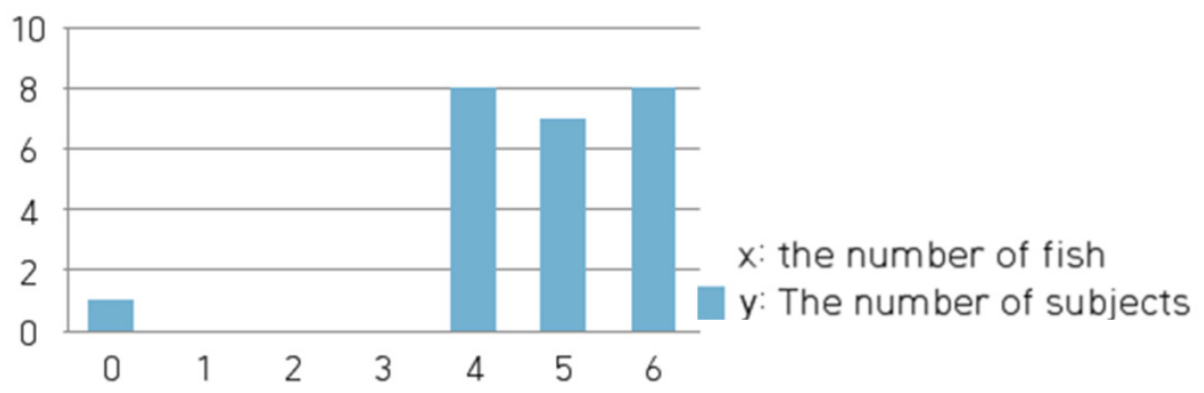

Fig. 6. Result of experiment. Limit time is 2 minutes.

\subsection{Refinements of System}

Color Notification. We collected qualitative data form questionnaire, comments and observations. During semi-constructed interviews, we could get some comments. Some subjects said visual superimposing of real and virtual content in 3D space creates confusion about distinction. They cannot recognize where to interact. So, we made the guidance that presented information about whether user interacts or not. For this purpose, we considered a possible solution by providing color change of augmented objects. As Fig.7 shows, if user catch augmented fish, fish color is changed.

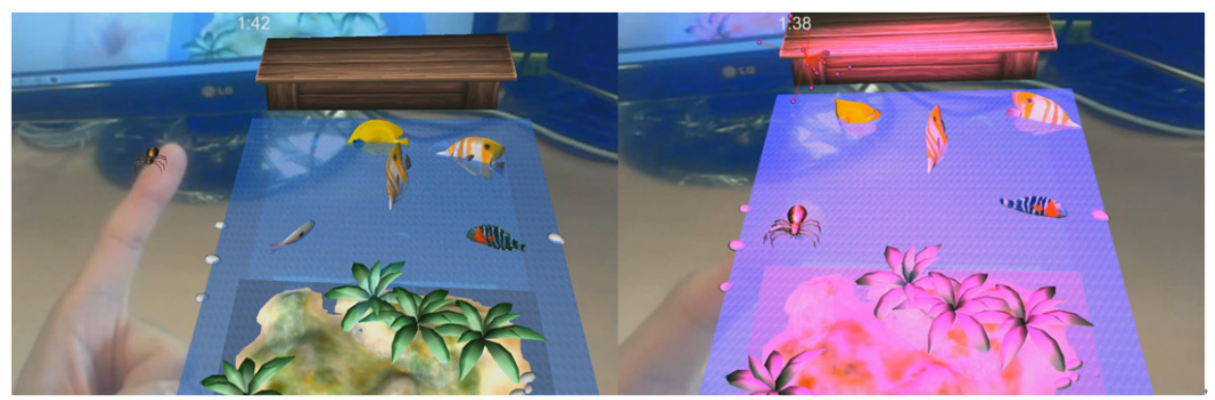

Fig. 7. Color is changed if a fish is caught 
Reducing Size. For tuning the degree of difficulty, we shortened the length, height and width of augmented fish by 0.18 times. After lessening the size of fish, people tend to take longer time to catch a fish. The result is shown in Fig. 8. Subjects caught 2.3 fish averagely.

Table 1. Change the size of fish and result of experiment. We scaled down the game object, fish. Modified fish size is one fifth than original one. Subjects try to catch the fish averagely 3 times. It is longer than before by 3times.

\begin{tabular}{|l|l|l|}
\hline & Before & After \\
\hline Fish scale & $\mathrm{x}=0.2 \mathrm{y}=0.4 \mathrm{z}=1$ & $\mathrm{x}=0.05 \mathrm{y}=0.05 \mathrm{z}=0.2$ \\
\hline the number of times trying & 1.1 & 3 \\
\hline
\end{tabular}

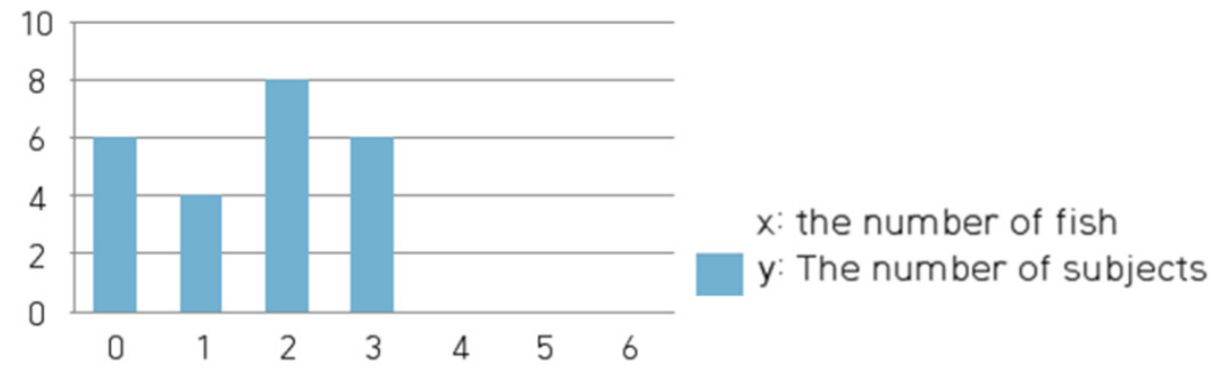

Fig. 8. After scale down, result of experiment

\section{Conclusion and Discussion}

In this paper, we introduce AR fishing game for enhancing user immersion and overcoming space limit. Interacting augmented objects with human hands in our system will strength the basis of how to control games. Our method is more powerful than the conventional immovable-video game using cumbersome equipment and mobile game. Our AR fishing game method can be applicable to many AR applications (i.e., future experimental education, urban planning, military simulation, collaborative surgery, etc.).

The techniques we described could be applied to other sports simulation. In order to apply the techniques to other sports simulation, different and sophisticated interaction techniques should be explored and studied in more detail. The project also shows how interaction techniques in $3 \mathrm{D}$ environment can be used to create an entertainment.

\section{Future Work}

For refining our design environment, different interaction techniques would be explored and studied in more detail. For example, if user takes a natural posture of 
throwing a fishing rod to catch fish, this gesture will be implemented as direct input on the system. Also, waving hand controls how far the fishing rod casts.

For future work, we plan to use RGB-D Image capturing including color and depth information together for modeling and tracking. It makes players more focus on game with their bodies than playing traditional game. Moreover, we make augmented fish movement more realistic in specific situation and develop tracking to enable users to have a continuous experience whether or not the target remains visible in the HMD field of view. We investigate the applicability of the developmental concepts to other sports simulation or game.

\section{References}

1. Kim, N., Yoo, W., Lee, Y., Chung, S., Han, M., Yeo, W.: Designing Intuitive Spatial Game using Brain Computer Interface. In: KHCI 2009, p. 1160 (2009)

2. Ionescu, D., Ionescu, B., Gadea, C., Islam, S.: A Multimodal Interaction Method that Combines Gestures and Physical Game Controllers. In: 2011 Proceedings of 20th International Conference on Computer Communications and Networks (ICCCN), pp. 1-6 (2011)

3. Koh, R.K.C., Duh, H.B.-L.: An integrated design flow in user interface and interaction for enhancing mobile AR gaming experiences. In: 2010 IEEE International Symposium on Mixed and Augmented Reality - Arts, Media, and Humanities (ISMAR-AMH), pp. 47,52 (2010)

4. Noh, K.: A Structural Equation Modelling of the Relationship between User Experience. Self-Efficacy and Game Performance in Healthcare Serious Game, Korea Game Society $12,15-20(2012)$

5. Lou, Y., Wu, W., Zhang, H., Zhang, H., Chen, Y.: A Multi-User Interaction System Based on Kinect and Wii Remote. In: 2012 IEEE International Conference on Multimedia and Expo Workshops (ICMEW), pp. 667,667 (2012)

6. Shires, L., Battersby, S., Lewis, J., Brown, D., Sherkat, N., Standen, P.: Enhancing the tracking capabilities of the Microsoft Kinect for stroke rehabilitation. In: 2013 IEEE 2nd International Conference on Serious Games and Applications for Health (SeGAH), pp. 1,8 (2013)

7. Zeng, M., Liu, Z., Meng, Q., Bai, Z., Jia, H.: Motion capture and reconstruction based on depth information using Kinect. In: 2012 5th International Congress Image and Signal Processing (CISP), pp. 1381,1385 (2012)

8. Kronrod, A., Danziger, S.: "Wii Will Rock You!" The Use and Effect of Figurative Language in Consumer Reviews of Hedonic and Utilitarian Consumption. Journal of Consumer Research 40(4), 726-739 (2013)

9. Foxlin, E., Harrington, M., Pfeifer, G.: Constellation: A wide range wireless motion tracking system for augmented Reality and Virtual set application. In: Studies in Proceedings of Siggraph 1998 Computer Graphics Proceedings. ACM SIGGRAPH Conference Series (1998)

10. Vasiljevic, T.P.: Fishing spear gun with dual spear projecting means. U.S. Patent (3), 340,642 (1967)

11. Chen, F.-S., Fu, C.-M., Huang, C.-L.: Hand gesture recognition using a real-time tracking method and hidden Markov models. Image and Vision Computing 21(8), 745-758 (2003) 
12. Qualcomm Vuforia, https://developer.vuforia.com/resources/devguide/image-targets

13. Black, J., Ellis, T.: Multi camera image tracking. Image and Vision Computing 24(11), 1256-1267 (2006)

14. Rehg, J.M., Kanade, T.: Visual tracking of high DOF articulated structures: An application to human hand tracking. In: Eklundh, J.-O. (ed.) ECCV 1994. LNCS, vol. 801, pp. 35-46. Springer, Heidelberg (1994) 